\title{
Platelet Rich Plasma in Androgenetic Alopecia- A Prospective Study
}

\author{
Mary Vineetha ${ }^{1}$, Seena Palakkal2 ${ }^{2}$, Jaya K. V. ${ }^{3}$ \\ ${ }^{1}$ Department of Dermatology, Government Medical College, Kottayam, Kerala, India. ${ }^{2}$ Department \\ of Dermatology, Government Medical College, Kottayam, Kerala, India. ${ }^{3}$ Department of \\ Community Medicine, Government Medical College, Thrissur, Kerala, India.
}

\section{ABSTRACT}

\section{BACKGROUND}

Androgenetic alopecia (AGA) is a common cause of hair loss in males and this has profound effect on the quality of life of patients. There are various treatment modalities and Platelet Rich Plasma (PRP) is one with promising effect in various studies. It contains platelets at a concentration of more than one million and contains various growth factors such as platelet-derived growth factors, vascular endothelial growth factors, epidermal growth factors which promote hair growth. As it is autologous, it has very few side effects.

\section{METHODS}

20 patients with AGA belonging to the age group 18- 40 years, Hamilton Norwood Stage 1-4 who attended dermatology OPD were included. Dermoscopic analysis of hair count was done at a specific distance from left medial eyebrow; the hair density was noted; images were taken and recorded. PRP was prepared by Manual Double Spin method; Under local anaesthesia, both spray technique and microdermabrasion injection technique were done. The procedure was repeated every 3 months and dermoscopy was done at each visit at the fixed site. Statistical analysis was done with ANOVA test and paired t test for these groups.

\section{RESULTS}

$83 \%$ had decrease in hair fall and $72 \%$ reported increase in hair growth at 3 months. Pain was the most common side effect. There was significant increase in hair density at each visit and there was negative correlation between duration of disease and increase in hair density.

\section{CONCLUSIONS}

PRP is an effective method for treating AGA.

\section{KEY WORDS}

Androgenetic Alopecia, Platelet Rich Plasma, Manual Double Spin Method, Dermoscopy
Corresponding Author:

Dr. Seena Palakkal,

Associate Professor

Department of Dermatology,

Government Medical College,

Kottayam, Kerala, India.

E-mail: drmaryvineetha@gmail.com

DOI: $10.14260 / j e m d s / 2020 / 373$

Financial or Other Competing Interests: None.

How to Cite This Article:

Vineetha M, Palakkal S, Jaya KV. Platelet rich plasma in androgenetic alopecia- a prospective study. J. Evolution Med. Dent. Sci. 2020;9(22):1696-1699, DOI: $10.14260 / \mathrm{jemds} / 2020 / 373$

Submission 25-03-2020,

Peer Review 09-05-2020

Acceptance 15-05-2020,

Published 01-06-2020.

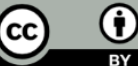




\section{BACKGROUND}

Androgenetic alopecia (AGA) is one of the commonest cases encountered by a dermatologist. It can have significant impact on quality of life of patients. ${ }^{1}$ Recent studies show association between AGA and metabolic syndrome. ${ }^{2}$ There has been tremendous increase in our knowledge regarding pathomechanisms leading to AGA and this has resulted in improved treatment outcome and better results. Stepwise miniaturization of the hair follicle leading to vellus transformation of terminal hair is the basis of development of AGA. $^{3}$ The terminal-to-vellus hair ratio is reduced from the normal 7:1 to $<4: 1$. Miniaturization of the follicles is followed by development of fibrous tracts. AGA is characterized by a patterned hair loss. Dermoscopy show yellow dots and variable diameter of the hair shafts. ${ }^{3}$ Various treatment options for AGA include topical minoxidil, systemic finasteride and hair transplantations. Newer modalities are under investigation. Platelet-rich plasma (PRP) is known to promote hair growth since 1993. ${ }^{4}$ PRP contains a large array of growth factors such as platelet-derived growth factors, vascular endothelial growth factors, epidermal growth factors, fibroblast growth factor-2 and insulin-like growth factors which promote hair growth by inducing the follicular stem cells to shift from a dormant to an active state. ${ }^{5}$ PRP has gained popularity for AGA due to its autologous nature, minimal invasiveness, absence of major side effects, and more affordable cost compared with hair restoration surgery. The mean blood platelet level is $200,000 \pm 75,000 / \mu \mathrm{L}$. Although the PRP platelet count has not been optimized, a platelet concentration of more than 1 million/ $\mu \mathrm{L}$ (approximately four to seven times the mean levels) is generally regarded as the therapeutically effective concentration of PRP. 6,7Due to its autologous origin and minimally invasive collection technique, the risk of infection and immune rejection is minimized. Various studies have proven the efficacy of PRP. 8,9 Male and female patients have had positive results from PRP injections in AGA in terms of regrowth, increased hair density, and improved quality of life. Only few Indian studies are there in this regard and so this study was conducted to determine the efficacy of PRP in AGA.

\section{METHODS}

It was a prospective study including 20 cases of clinically diagnosed androgenetic alopecia (AGA) Hamilton Norwood (HN) stage 1-4 ${ }^{10}$ who attend the skin outpatient department from year 2015 to 2016 in the age group 18-40 years. Patients aged more than 40, higher Hamilton Norwood stage $>4$, patients with unrealistic expectations, history of seizures, blood dyscrasias and keloidal tendency were excluded. AGA was diagnosed by detailed history taking and clinical examination. History of previous treatment modalities and family history were noted. Routine blood examination, retro screening, HBsAg, Anti HCV antibody were done in all patients.

After obtaining informed consent patients were selected for PRP therapy. PRP was prepared by manual double spin method. ${ }^{11}$ For this $12 \mathrm{ml}$ venous blood is collected using $18 \mathrm{G}$ needle, $4 \mathrm{ml}$ each added to 3 autoclaved glass test tubes prefilled with $0.6 \mathrm{ml}$ of citrate phosphate dextrose (CPD) the anticoagulant. The test tubes are closed with sterile cotton, labelled and centrifuged at $1500 \mathrm{rpm}$ for 15 minutes. The buffy coat was carefully separated using $18 \mathrm{G}$ needle and syringe along with plasma and few RBC. This is transferred to another sterile test tube, which is closed with sterile cotton and centrifuged at $1500 \mathrm{rpm}$ for 10 minutes. Now the platelet rich plasma is seen at bottom and supernatant is platelet poor plasma. This need to be used immediately after discarding the $2 / 3$ of supernatant and resuspending to get uniform distribution of platelets throughout. This platelet rich plasma will contain around 4- 7 times the mean blood level of platelets. The centrifugation process separates blood components owing to their different specific gravities, i.e., RBCs being the heaviest, followed by WBCs, whereas platelets are the lightest. The first centrifugation is slow to avoid spinning down platelets and to isolate plasma. Platelets are mostly concentrated right on top of the buffy coat layer. Subsequent centrifugation is faster, so that platelets are spun down and separate as a pellet at the bottom of the tube from platelet-poor plasma (PPP) above. This Method is called differential centrifugation,

The patients are instructed to take shampoo bath using antifungal shampoo on the day of procedure. Dermoscopic examination will be done at a fixed point for each patient on scalp, on a line joining the medial aspect of left eyebrows and scalp and images will be recorded for analysing the results. Dermlite is the dermascope used. Topical anaesthetic will be applied on the scalp 30 minutes before procedure. Scalp is cleaned using betadine wash and saline. Parallel and perpendicular markings were made using marker pen for ease of injection and not to miss any points. Both spray technique and injection techniques were used in a patient. Dermaroller $1.5 \mathrm{~mm}$ rolled over scalp to produce minute bleeding points. PRP taken in insulin syringes will be sprayed over this and massaged for better penetration. Injection technique was also done $0.5 \mathrm{~mm}$ apart near hair follicles $2 \mathrm{~mm}$ deep approximately $0.1 \mathrm{ml}$ injected at each site. ${ }^{5}$

Following procedure sterile cap was used to cover the scalp, tetanus toxoid injections and antibiotics were given and patients instructed not to wash the scalp for 24 hours. Dermoscopic examinations were done before first sitting and before $2^{\text {nd }}$ and third sittings, which were at 3 months intervals.

\section{RESULTS}

Total of 20 patients were enrolled in study, average age was 28.2 years, age group ranged from 22-36 years. 12 patients were of HN grading 4 and 8 patients of grade 3. Duration of alopecia was less than 5 years in 13 and more than 5 years in 7 patients. Family history was there in 11 patients. 7 patients were on allopathic treatment and 3 patients were on indigenous medicines. Out of these 2 patients were using minoxidil. Hypertension and hypothyroidism were there in one patient. Average age of onset was 23.5 years and it ranged from 16- 29 years

Immediate side effect was pain in $85 \% .3$ patients developed occipital and post auricular lymphadenopathy and one patient developed fever and post auricular lymphadenopathy. $83 \%$ had decrease in hair fall and $72 \%$ reported increase in hair growth at 3 months. Mean hair 
density at 0,3 and $6^{\text {th }}$ month was calculated, and Repeated measures ANOVA test was done to find if the difference in means was statistically significant. Test value was $F=43.328$ with degree of freedom $\mathrm{df}=2$ and $\mathrm{p}$ value $<0.05$. So, the difference between the groups was statistically significant. (Table 1, Fig. 1) Paired t test was done to analyse the difference between 0 and $3^{\text {rd }}$ month and $3^{\text {rd }}$ and $6^{\text {th }}$ month, and this difference was also statistically significant with $\mathrm{p}$ value $<0.05$. (Table 2) In patients with grade 3 alopecia $62.5 \%$ had family history and patients with grade 4 alopecia $50 \%$ had family history. Pearson correlation test was done to find correlation between duration of illness and hair density, Pearson correlation quotient was -.386 which shows negative relation between duration of illness and increase in hair growth.

\begin{tabular}{|ccccc|}
\hline Month of Therapy & Mean Hair Density & S.D. & F & P Value \\
Hair density 0 & 19.95 & 7.522 & 43.328 & .000 \\
Hair density 3 & 29.10 & 8.309 & 43.328 & .000 \\
Hair density 6 & 34.80 & 6.779 & 43.328 & .000 \\
Test value is F = 43.328 with degrees of freedom (df) = 2, \\
P value < 0.05. So, the difference between groups is statistically significant \\
\hline Table 1. Means of Three Different Groups at 0, 3 and 6 Months \\
\hline
\end{tabular}

\begin{tabular}{|ccccc|}
\hline $\begin{array}{c}\text { (I) } \\
\text { Month Difference }\end{array}$ & $\begin{array}{c}\text { Measure: Measure_1 } \\
\text { Difference }\end{array}$ & $\begin{array}{c}\text { Mean } \\
\text { Difference (I-J) }\end{array}$ & $\begin{array}{c}\text { Std. } \\
\text { Error }\end{array}$ & Sig. $^{\text {a }}$ \\
1 & 2 & $-9.150^{*}$ & 1.800 & .000 \\
& 3 & $-14.850^{*}$ & 1.780 & .000 \\
2 & 1 & $9.150^{*}$ & 1.800 & .000 \\
& 3 & $-5.700^{*}$ & 1.168 & .000 \\
3 & 1 & $14.850^{*}$ & 1.780 & .000 \\
& 2 & $5.700^{*}$ & 1.168 & .000 \\
\hline \multicolumn{4}{|c|}{ Table 2. Pairwise Comparisons at Different Months } \\
\hline In all groups the difference of mean is statistically significant p value is $<0.05$ \\
\hline
\end{tabular}

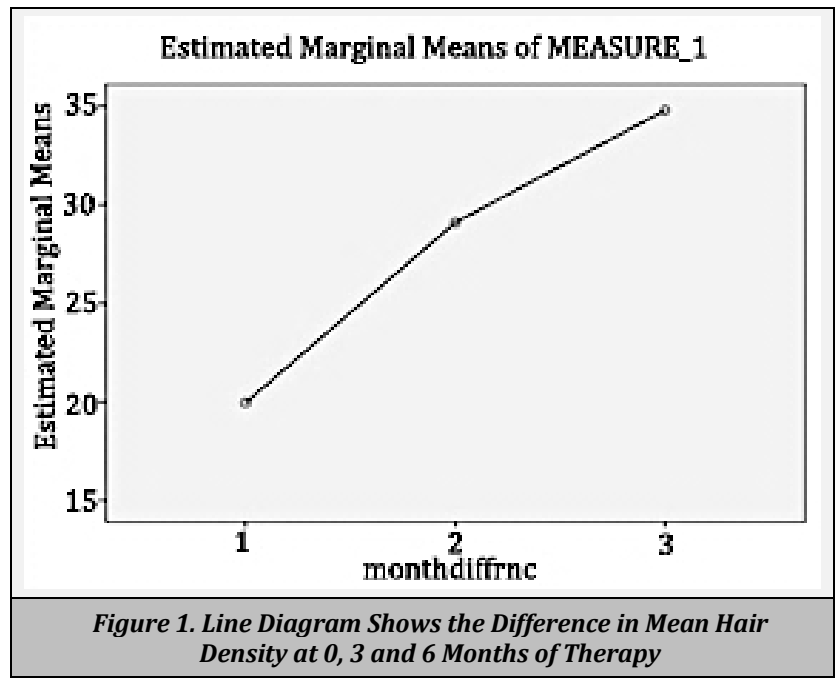

\section{DISCUSSION}

Androgenetic alopecia (AGA) or pattern alopecia, is a common condition occurring in males with immense psychological impact in a few irrespective of age or stage of baldness.

It can be a predictor of premature initiation of severe coronary heart disease and metabolic syndrome. In AGA there is a gradual conversion of terminal hairs into indeterminate, and finally into vellus hairs. PRP is an autologous preparation of plasma with a high concentration of platelets. It contains various growth factors and cytokines with inherent capacity to repair and regenerate. It is useful in the treatment of acne scars, non-healing wounds, hair loss, and enhancing fat graft survival.

The expert panel for management of AGA says that that patient selection is crucial in PRP; the procedure should not be prescribed for all patients with AGA.12 PRP is used as a holding solution for the grafts in hair transplant. The panel recommended that PRP should not be used as monotherapy, but as an adjuvant to minoxidil/finasteride. The mechanism of PRP therapy involves proliferation of dermal papilla cells induced by the growth factors of the activated PRP. The main disadvantages of PRP are pain during the procedure and there is no clarity regarding the number of sessions and interval between sessions due to reversible effects.

Mean age of our patients was 28.2 years which was in concordance with study conducted by Gupta et al $^{13}$ and Sharma et al. ${ }^{14}$ But in Gkini et al study it was 34 years. Average age of onset was 23.5 years which was also in concordance with Gupta et al study. Family history was seen in $55 \%$, but in Gupta et al study it was $87 \%$. In Gikini et al ${ }^{9}$ study all patients had pain but was $85 \%$ in our study. Fever and post auricular lymphadenopathy is not reported in other studies.

$72 \%$ reported increase in hair growth and it was $66 \%$ in Gupta et al study. $83 \%$ had decrease in hair fall and it was 93 $\%$ in Gupta et al study. There was significant increase in hair density after each sessions and paired t test was done to assess difference between various groups which was significant ( $p$ value $<0.05$ ).This was in concordance with studies by Lopez et $\mathrm{al}^{15}$ and Greco et al ${ }^{16}$. Mean hair density was $19.95+/{ }_{-} 7.552$ before procedure and $29.10+/-8.309$ at $3^{\text {rd }}$ month and $34.80+/-6.779$ at $6^{\text {th }}$ month. Repeated ANOVA test showed that this difference was significant with $\mathrm{p}$ value $<0.05$. Negative relation between duration of illness and increase in hair density was found in our study which was demonstrated in studies by Gupta et al. ${ }^{13}$

\section{CONCLUSIONS}

PRP is an effective treatment modality for AGA with decrease in hair fall and increase in hair density. As it is autologous it is devoid of major side effects and pain is the only major side effect noticed. As the duration of disease increases the response to therapy decreases. Hence PRP can be considered as an adjunct therapy in early cases of AGA.

\section{REFERENCES}

[1] Sehgal VN, Kak R, Aggarwal A, et al. Male pattern androgenetic alopecia in an Indian context: a perspective study. J Eur Acad Dermatol Venereol 2007;21(4):473-9.

[2] Gopinath H, Upadya GM. Metabolic syndrome in androgenic alopecia. Indian J Dermatol Venereol Leprol 2016;82(4):404-8.

[3] Kaliyadan F, Nambiar A, Vijayaraghavan S. Androgenetic alopecia: an update. Indian J Dermatol Venereol Leprol 2013;79(5):613-25.

[4] Knighton DR. Inventor: Curative Technologies, Inc., assignee. Method for promoting hair growth. United States patent 5178883. Jan 12, 1993. 
[5] SendhilKumaran AM. Platelet-rich plasma in dermatology: boon or a bane? Indian J Dermatol Venereol Leprol 2014;80(1):5-14.

[6] Li ZJ, Choi HI, Choi DK, et al. Autologous platelet-rich plasma: a potential therapeutic tool for promoting hair growth. Dermatol Surg 2012;38(7 Pt 1):1040-6.

[7] Weibrich G, Kleis WKG, Hafner G. Growth factor levels in the platelet-rich plasma produced by 2 different methods: Curasan-type PRP kit versus PCCS PRP system. Int J Oral Maxillofac Implants 2002;17(2):184-90.

[8] Singhal P, Agarwal S, Dhot PS, et al. Efficacy of plateletrich plasma in treatment of androgenic alopecia. Asian J Transfus Sci 2015;9(2):159-62.

[9] Gkini MA, Kouskoukis AE, Tripsianis G, et al. Study of platelet-rich plasma injections in the treatment of androgenetic alopecia through an year period. J Cutan Aesthet Surg 2014;7(4):213-9.

[10] Hamilton JB. Patterned loss of hair in man: types and incidence. Ann N Y Acad Sci 1951;53(3):708-28.
[11] Gonshor A. Technique for producing platelet-rich plasma and platelet concentrate: background and process. Int J Periodontics Restorative Dent 2002;22(6):547-57.

[12] Mysore V, Parthasaradhi A, Kharkar RD, et al. Expert consensus on the management of androgenetic alopecia in India. Int J Trichol 2019;11(3):101-6.

[13] Gupta S, Revathi TN, Sacchidanand S, et al. A study of the efficacy of platelet-rich plasma in the treatment of androgenetic alopecia in males. Indian J Dermatol Venereol Leprol 2017;83(3):412.

[14] Sharma VK, Bhari N, Patra S, et al. Platelet-rich plasma therapy for androgenetic alopecia. Indian J Dermatol 2019;64(5):417-9.

[15] Lopez V, Vaya A, Bautista D, et al. Autologous platelet-rich plasma as a potential therapeutic tool in androgenetic alopecia. J Am Acad Dermatol 2013;68(Suppl 4):AB103.

[16] Greco J, Brandt R. The effects of autologous platelet rich plasma and various growth factors on non-transplanted miniaturized hair. Hair Transplant Forum Int 2009;19:4950 . 